Journal of Environmental Science and Public Health

doi: $10.26502 /$ jesph. 96120028

Volume 2, Issue 1

Research Article

\title{
Using DNA Barcoding Technique to Identify Some Scleractinian Coral Species along the Egyptian Coast of the Red Sea and Southern of Arabian Gulf
} \author{
Hasan Hanafy ${ }^{2}$ \\ ${ }^{1}$ Department of Marine Science, Faculty of Science, Port Said University, Port Said, Egypt \\ ${ }^{2}$ Department Marine Science, Faculty of Science, Suez Canal University, Ismailia, Egypt
}

Mohammed Ahmed Sadek ${ }^{1^{*}}$, Mohammed Ismail Ahmed ${ }^{2}$, Fedekar Fadel Madkour ${ }^{1}$ and Mahmoud

"Corresponding Author: Mohammed Ahmed Sadek, Department of Marine Science, Faculty of Science, Port Said University, Port Said, Egypt, Tel: 00971501092572; E-mail: kamel.1187@gmail.com

Received: 05 February 2018; Accepted: 22 February 2018; Published: 28 February 2018

\begin{abstract}
Scleractinian corals Phylogenetic studies have been shown that most taxa are not matched with their evolutionary histories. Using gross morphology as principal base, traditional taxonomy cannot solve the lack of well-defined and homologous characters that can describe scleractinian diversity sufficient. Scleractinian Coral species are hard to identify because of their morphological plasticity. DNA barcoding techniques were used to build molecular phylogenetic analysis to some common scleractinian species in Red Sea and Arabian Gulf. The phylogenetic analysis showed that Porites harrisoni, was clustered separate from other Porites sp collected from gene bank. While for Acropora digitefra genetic divergences were recorded between samples collected from the Red Sea and Gulf of Aqaba. Platygyra daeleda sample from Fanous (Red Sea) were distinct from other Platygyra daeleda samples same case were recorded with Pocillopora verrucosa samples. The DNA barcoding technique proves to be very useful in not only differentiating between species but also finding genetic diversity within species.
\end{abstract}

Keywords: Scleractinian corals; Phylogenetic; COXI; DNA barcoding 


\section{Introduction}

Morphological identification is confronted with difficulties from phenotypic plasticity, life stage, gender; inability to identify cryptic species, animal parts and lack of taxon knowledge [1]. Resolving many long-standing problems in traditional systematics come from its reliance on skeletal macromorphology, Modern coral taxonomy has begun to integrate examinations of colony, corallite, and subcorallite morphologies with the molecular sequence data that have been collected in the last decade.

Many taxa spread across the scleractinian tree of life have been incorporated into a rigorous classification underpinned by greater phylogenetic understanding [2]. Morphological and physiological diverse within scleractinian corals species [3-5] suggests that their population structure is complex [5]. The complexity of coral population structures has been some illustration by Allozyme electrophoresis [6-8], but it was limited by number of loci and the level of variation at these loci. Using DNA-based techniques recently develop our understanding of the genetic structure of coral populations [9].

DNA barcoding is a taxonomic method to identify the particular taxon of organism that uses a short genetic marker in its DNA [10]. A lot of applications of DNA barcoding in animals had been used a 658-bp region of the mitochondrial (mt) cytochrome c oxidase subunit I (COI) gene widely [10, 11]. However, the rate of evolution in mitochondrial genomes is slow which is usefulness of COI barcoding for identifying coral species specially anthozoans in general $[12,13]$. On another hand, across wide range of phylogenetic analyses of scleractinian species showed that COI barcoding technique is consistent with coral taxonomy to level of genus $[14,15]$, and also to level of species in Stylophora [16]. All of these results make that COI can be a genetic tool for expand taxonomic resolution of corals. On another hand, the advantage of using COI sequence data for coral phylogeny is that, unlike the $16 \mathrm{~S}$ rDNA, 12S rDNA, and 28S rDNA genes, the sequences are unambiguously alignable because they contain no indels [15].

Aim of study is to investigate molecular characteristic of selected reef building corals collected from the Red Sea and the Arabian Gulf to understand the lineage between scleractinian coral species in such geographic region.

\section{Materials and Methods}

\subsection{Sample collection}

Specimens of scleractinian corals (Five branched corals namely, Acropora digitifera, Acropora humilis, Acropora pharaonis, Pocillopora verrucosa, and Stylophora pistillata in addition to three massive corals namely, Platygyra deadalea, Porites harrisoni, and Favia pallida were collected during the period between January 2012 and March 2013 by SCUBA diving following PADI instruction. 


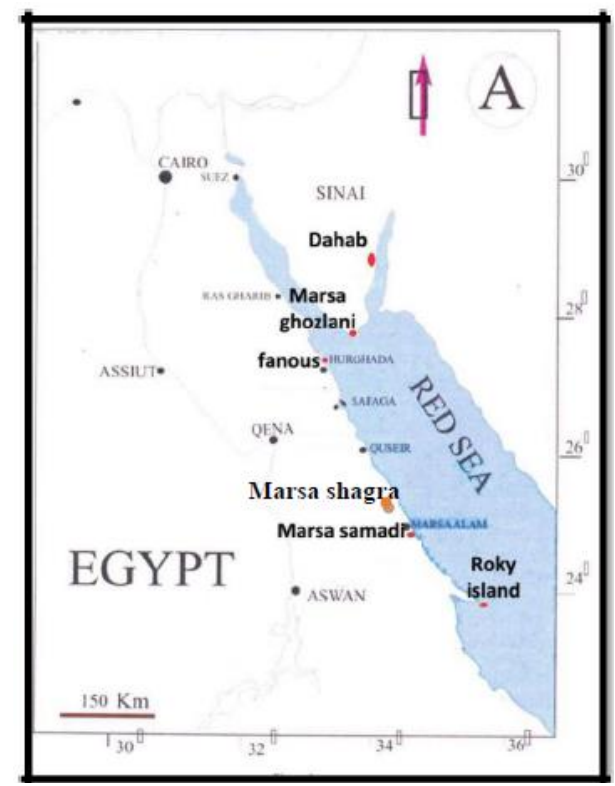

Figure 1: Map showing coral sampling and survey sites (red dots) at the Egyptian coast of Red Sea.

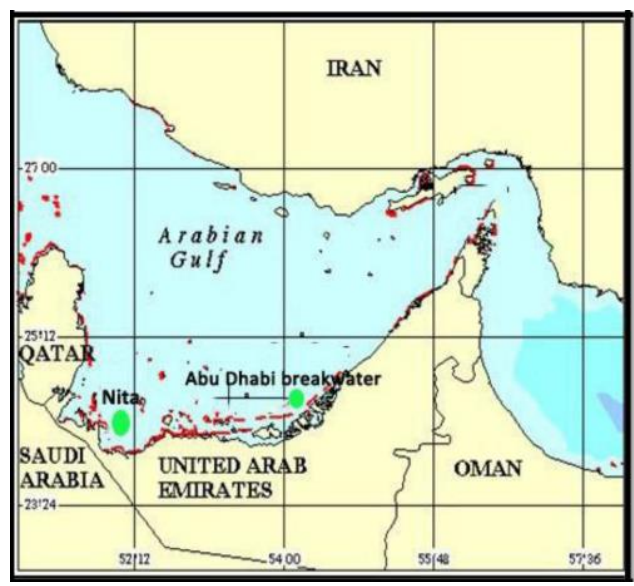

Figure 2: Map showing coral sampling and survey sites (green dots) at the southern coast of Arabian Gulf.

Small fragments of the branched corals were collected randomly by wrapping central branches with pre-labeled sterile plastic bags and cutting nearly $7 \mathrm{~cm}$ length by clipper. While massive species were collected by hammer and chisel. To avoid re-sampling coral fragments of the same genus, the distance between replicates were more than $5 \mathrm{~m}$. Specimens were identified using field recognizable features (colony life form, color and corallites form, etc.) according to Veron et al. [17], then later, confirmed by analysis of skeletal traits in the laboratory. Identification of samples was confirmed by M. Pichon (personal communication).

Specimens collected from southern Arabian Gulf were preserved in 95\% ethanol while the specimens collected from Egyptian coasts of the Red Sea were preserved in ice or dimethyl sulfoxide (DMSO) buffer until arrive the 
laboratory. Coral tissues were separated for DNA analysis. The skeleton was bleached in 5ppt sodium hypochlorite, and the specimens were let to dry.

\subsection{DNA extraction, amplification and sequencing}

DNA extrication had been done by Phenol/Chloroform Extraction Method (PC). Purity of gDNA (values of 260/280 and 260/230 ratios) was checked using NanoDrop. Agarose gel electrophoresis was used to detect quality of DNA. A region of the mitochondrial cytochrome oxidase I (COI) gene was amplified using specific primers for Scleractinia. The primer set was composed of the forward one MCOIF (5' TCT ACA AAT CAT AAA GAC ATA GG 3') and the other reverse MCOIR (5' GAG AAATTA TAC CAA AAC CAG G 3') [18]. Successfully amplified fragments of COXI gene were purified with QIAquick PCR purification kit (QIAGEN) then sequenced in the forward and reverse direction using BigDye Terminator V3.1 Cycle Sequencing Kit and run on 3500 Genetic Analyzer, Applied Biosystems in Biotechnology Research Center, Suez Canal University.

Chromatograms of COXI were edited by using MEGA V6.06 software and aligned with the Clustal W version 1.6 [19]. Sequences were adjusted manually and then were blasted on NCBI GenBank under default algorithm parameters. All COI edited sequences of the current study were deposited at NCBI GenBank with accession numbers from KR401093 to KR401104.

\subsection{Data analysis}

Reconstruction of phylogenetic trees was based on 1000 bootstrap replication value. All phylogenetic analyses were conducted using MEGA6 [20]. Based on maximum likelihood method, phylogenetic relationship between nucleotide sequences of COX1 on the current study was inferred using Hasegawa-Kishino-Yano substitution model and Gamma distributed among sites (HKY $+\mathrm{G})$ according to the lowest Bayesain Information Criterion (BIC), to construct tree also tree with their relative's species from GenBank that rooted by Sacrophyton sp.

\section{Results}

Based on DNA quality and quantity and PCR successfulness (see Figure 1 for example), only 12 samples represent 5 species of common scleractinian corals were selected for COI barcoding. The tissues of scleractinian corals contain the genomes of intracellular symbiotic dinoflagellates (zooxanthellae). This non-coral DNA can cause significant contamination in any attempt to isolate or amplify coral DNA. Previously, this problem was overcomed either by using zooxanthella-free coral gametes [21-23] or by the repeated centrifugation of adult coral tissues to separate hard coral tissue and zooxanthellae [24]. However, the first method is stated that come coral species only spawn annually and other species brood and release planula larvae that contain zooxanthellae [25] or have gametes that already contain zooxanthellae [26]. While the most zooxanthellae exclude in the second method, this does not guarantee complete absence of the zooxanthellae's nucleic acid, which cause problem for sensitive techniques such as the polymerase chain reaction (PCR). 


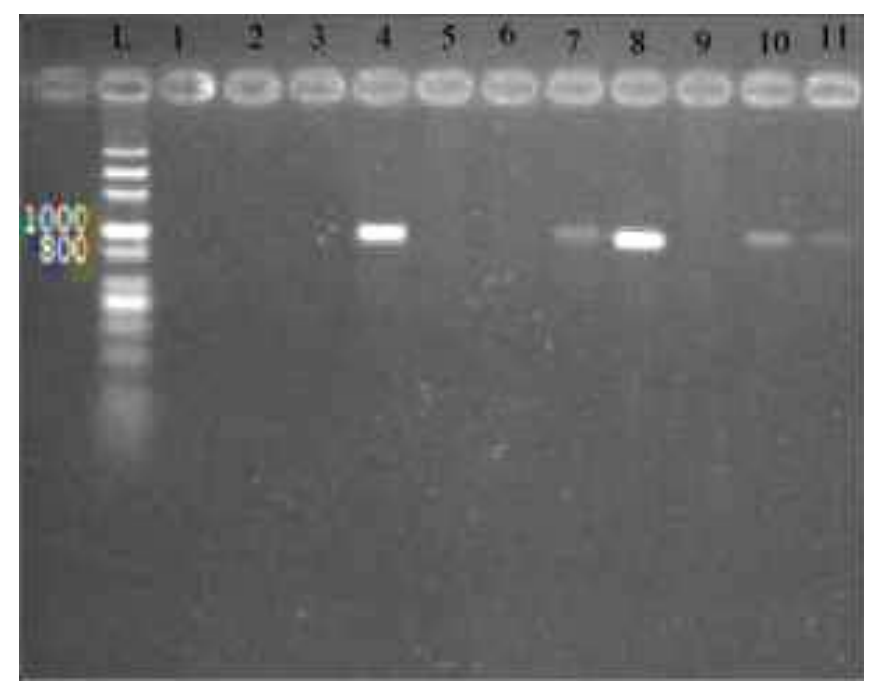

Figure 3: 1.5\% agarose gel electrophoresis of COI amplicons of Acropora digitifera (lane 4), Favia pallida (lane 7), Pocillopora verrucosa (lane 8), Platygyra daedalea (lane10), and Porites harrisoni (lane11). L; Molecular size ladder of 100-3000 bps.

The ML phylogenetic analysis (Figure 5) showed two major distinct clades. Acropora spp. clustered in one clade while the remaining species clustered in one clade (with a highbootstrap values of 99\%). However, the second clade was further subdivided into two subclades; one for Pocillopora sp. (with bootstrap value of 100\%) and the other for Porites, Favia and Platygyra (with bootstrap value of 99\%).

For Acropora sp. of the present samples (Acropora digitefera) was clustered with another Acropora sp. also, formed one group. While Acropora digitefera from Taiwan nears to it but not clustered in the group (Figure 4A). The ML tree showed that samples (Favia pallida Fanous, Nita) clustered with Favia pallida from Japan with high bootstrap value (85\%) (Figure 4B). The ML tree showed Platygyra daedalea from Nita and Abu Dhabi breakwater was clustered with Platygyra daedalea from Yemen while Platygyra daedalea from Fanous clustered alone (Figure 4C).

The ML tree showed Pocillopora verrucosa (Fanous) clustered with Pocillopora verrucosa (Japan and Australia) and Pocillopora damicroinis (USA) (Figure 4D). The ML tree showed Porites harrisoni (Nita, Abu Dhabi) clustered together in one branch (with bootstrap 100\%) while other Porties species clustered in another branch with bootstrap (100\%) (Figure 4E). 


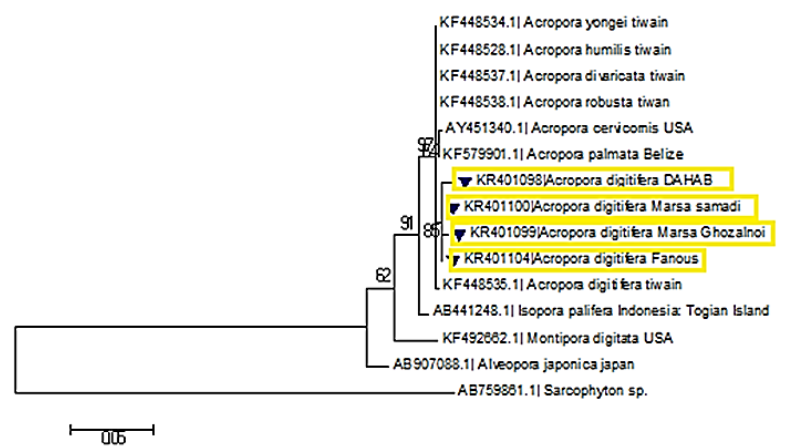

एण

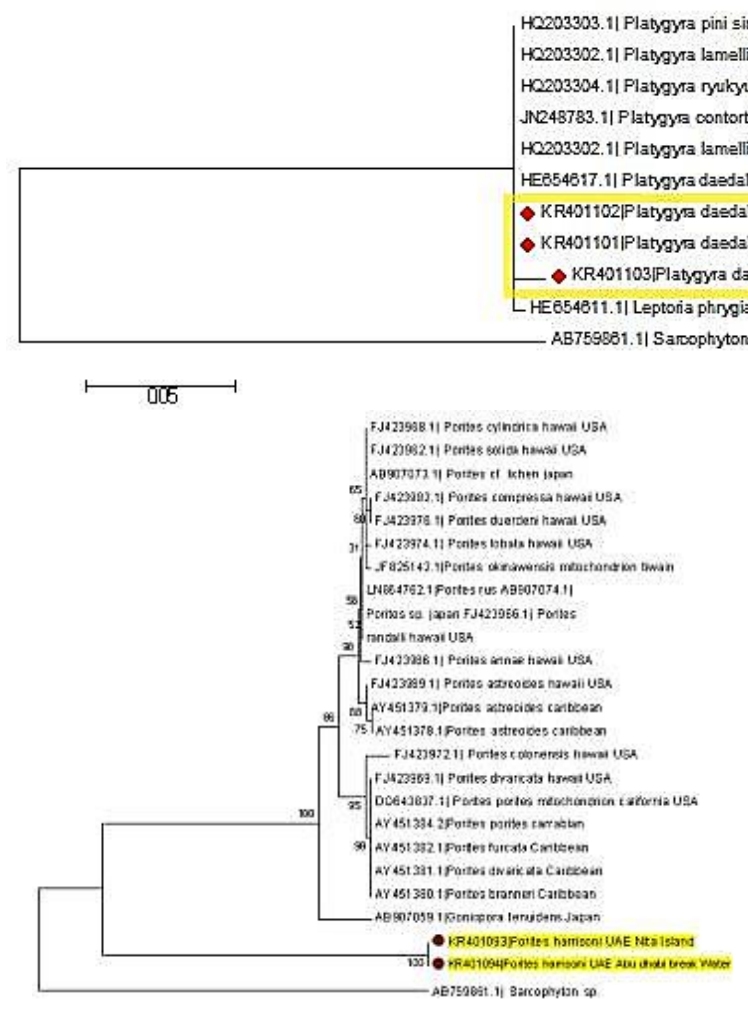

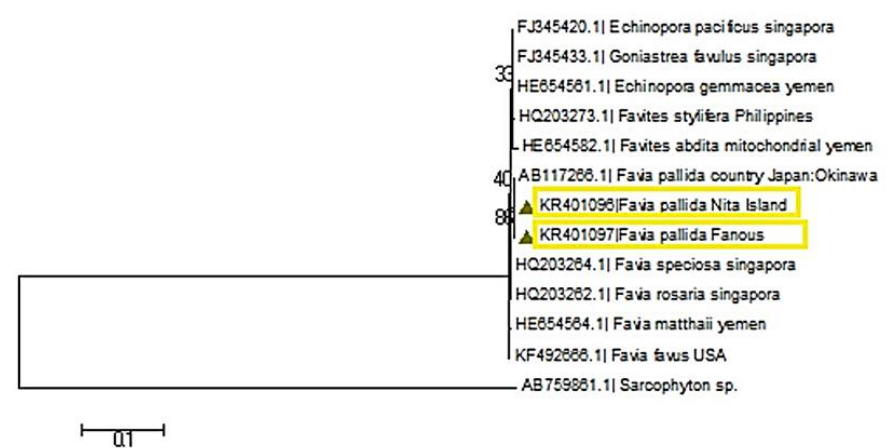

EF528303.1 Poeillopora eydowx ab:EU450213.1 Pocilopora daricomis tiwain AB441230.11 Pocilopora vervuossa Japan: Okinsas Aka Istand AY139812.11 Pocilopora eervucosa Australin a KR431095 Pocilipora vervoosa Fanous

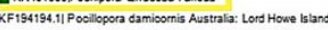
Gf EF633515,11 Seriatopora hystix Tha iland: Similan Island Andaman Sea 01 8759891.11 Saroophyton sp.

Figure 4: A) Maximum like-hood tree for COXI sequences of genus Acropora sp., B) Maximum like-hood tree for COX1 sequences of genus Favia and related genus., C) Maximum like hood tree for COXI sequences of genus Platygyra and related genera., D) Maximum like hood tree for COXI sequences of genus Pocillopora and related genera., E) Maximum like hood tree for COXI sequences of genus Porites and related genera. 


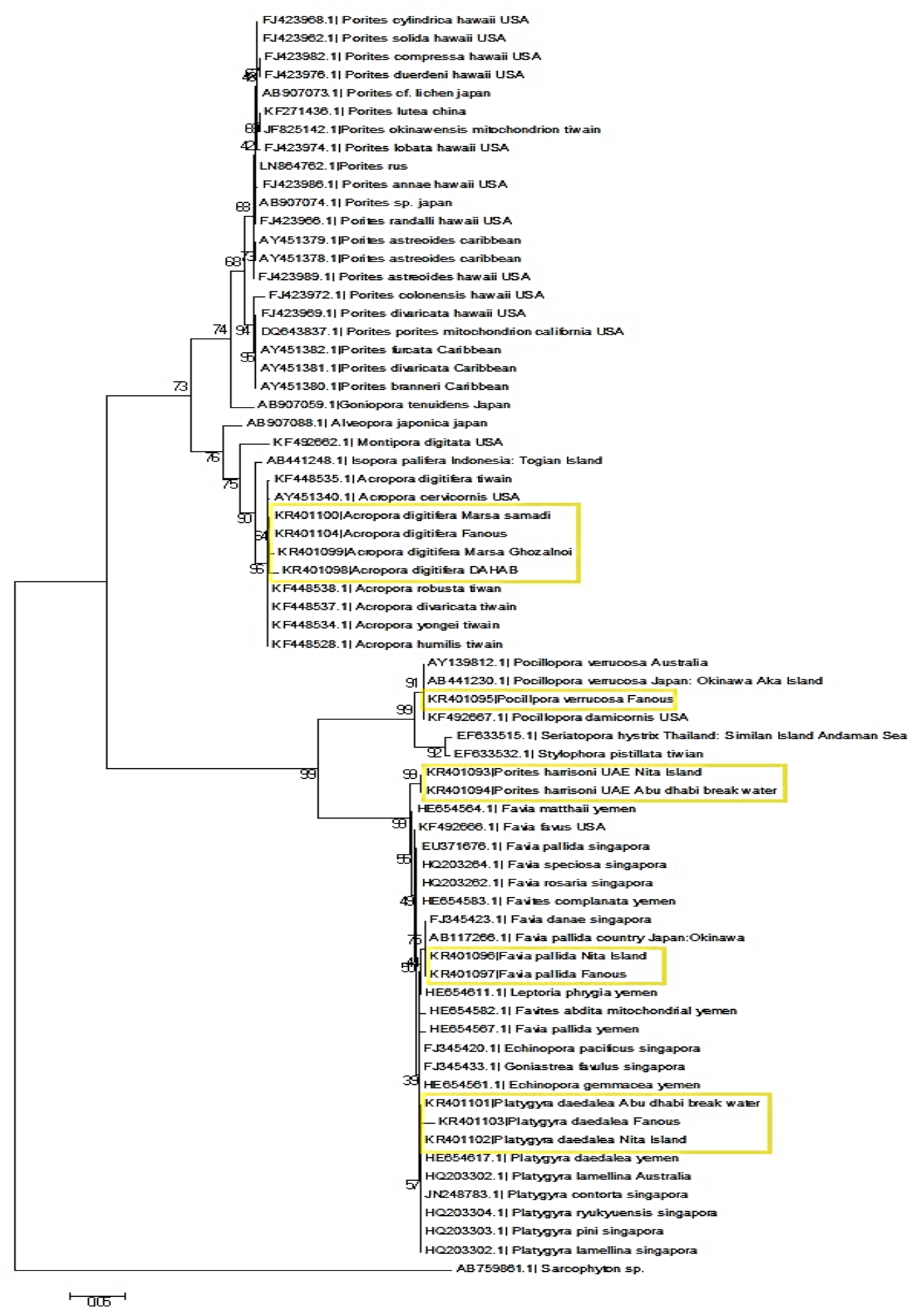

Figure 5: Maximum like-hood tree for COXI sequences of current study coral species and

GenBank sequences. 


\section{Discussion}

Phylogenetic analysis of specimens had been collected from sites and gene bank using DNA barcoding (COXI) was successful, and well support that scleractinia phylogenetic tree divided to two major clades complex as (Agariciidae, Acroporidae, Poritidae, Dendrophylliidae) and robust as (Pocilloporidae, Stenocyathidae, Faviidae, Fungiidae, Mussidae)'corals [10, 27, 28].

Phylogenetic analysis supports that genus Alveopora is grouped and well supported acroporidae clade with bootstrap (76\%). Based on the genetic analyses Alveopora transferred from poritidae to the acroporidae [29, 30]. At genus level, all specimens and that had been collected from gene bank support that Acropora is related to Isopora than Montipora with bootstrap (75\%). This is identical to the phylogenetic analysis to family acroporidae in level of genus as Anacropora is more related to Montipora, and Acropora to Isopora that was elevated to genus level recently [15]. Phylogenetic analysis of A. digitifera, $P$. verrucosa, and F. pallida indicates that there is no distinct sequence divergence between sits using COI molecular barcoding technique. In spite of being similar in their morphological characters, A. digitifera COI sequences in the current study and that in Taiwan showed divergence with $85 \%$ bootstrap percentage.

For the other coral species, the current study indicated that there is no distinct phylogenetic difference between populations of corals in the Red Sea and that elsewhere. No genetic differentiation found by using the mORF marker to identify distinct mitochondrial lineages for P. verrucosa in Red Sea (Saudi Arabia). According to these results, $P$. verrucosa in the Red Sea seems to belong to a single panmictic population [31]. Following molecular results of Fukami et al. [18] and Fukami et al. [14], genus Favia is to be include Atlantic taxa from Favia only and other species are assigned to genus Dipastarea.

F. pallida from Fanous reef showed changes in morphology (no calice), so itmay be another morphotype with identical DNA sequences. Phylogenetic analysis of Porites showed that Porites harrisoni specimens clustered in one clade, other porites spp. with bootstrap (100\%). Clustered together on another clade with high genetic distance. Benzoni and Stefani [32] found that Porites fontanesii has a distinct species basal to, and well divergent from, one of the two main clades so far identified in Porites using rDNA phylogeny. Phylogenetic analysis of Platygyra showed that all P. deadalea samples clustered with other Platygyra sp. However, only P. deadalea (Fanous) showed genetic distance from other $P$. deadalea that may suggest the presence of cryptic species. Arabian Gulf specimens were close to $P$. deadalea (Yemen). Two morphotypes of $P$. deadalea had been found in Kenya, significant genetic differences between two morphotypes however, phylogenetic analysis of ITS sequences showed no evidence of sequence divergence between morphotypes [33]. P. deadalea displayed 4 distinct morphotypes on the Great Barrier Reef (GBR) [34].

In Egyptian Red Sea and southern Arabian Gulf, this is the first study of phylogenetic analysis to some hard scleractinian coral species. Further research is required on both the Red Sea and the Arabian Gulf in order to 
understand coral genetic diversity and most importantly corals resistance and resilience to climate change and global warming.

\section{References}

1. Hebert PD, Cywinska A, Ball SL. Biological identifications through DNA barcodes." Proceedings of the Royal Society of London B: Biological Sciences 270 (2003): 313-321.

2. Huang D, Benzoni F, Fukami H, et al. Taxonomic classification of the reef coral families Merulinidae, Montastraeidae, and Diploastraeidae (Cnidaria: Anthozoa: Scleractinia). Zoological Journal of the Linnean Society 171 (2014): 277-355.

3. Wallace C, Willis B. Systematics of the coral genus Acropora: implications of new biological findings for species concepts. Annual review of ecology and systematics (1994): 237-262.

4. Takabayashi M, Hoegh-Guldberg O. Ecological and physiological differences between two colour morphs of the coral Pocillopora damicornis. Marine Biology 123 (1995): 705-714.

5. Veron JEN. Corals in space and time: the biogeography and evolution of the Scleractinia, Cornell University Press (1995).

6. Stoddart JA. Genetical structure within populations of the coral Pocillopora damicornis. Marine Biology, 81 (1984): 19-30.

7. Willis BL, Ayre DJ. Asexual reproduction and genetic determination of growth form in the coral Pavona cactus: biochemical genetic and immunogenic evidence. Oecologia 65 (1985): 516-525.

8. Hellberg ME. Relationships between inferred levels of gene flow and geographic distance in a philopatric coral, Balanophyllia elegans. Evolution 48 (1994): 1829-1854.

9. Avise J, Avise JC. Molecular Markers, Natural History and Evolution. Chapman and Hall. Journal of Evolutionary Biology 7 (1993): 766-767.

10. Hebert PD, Ratnasingham S, de Waard JR. Barcoding animal life: cytochrome c oxidase subunit 1 divergences among closely related species. Proceedings of the Royal Society of London B: Biological Sciences 270 (2003b): S96-S99.

11. Ward RD, Holmes BH, O'HARA TD. DNA barcoding discriminates echinoderm species." Molecular Ecology Resources 8 (2008): 1202-1211.

12. van Oppen MJ, Willis BL, Miller DJ. Atypically low rate of cytochrome b evolution in the scleractinian coral genus Acropora. Proceedings of the Royal Society of London B: Biological Sciences 266 (1999): 179-183.

13. Shearer T, Coffroth M. Genetic identification of Caribbean scleractinian coral recruits at the Flower Garden Banks and the Florida Keys." Marine ecology. Progress series 306 (2006): 133-142.

14. Fukami H, Chen CA, Budd AF, et al. Mitochondrial and nuclear genes suggest that stony corals are monophyletic but most families of stony corals are not (Order Scleractinia, Class Anthozoa, Phylum Cnidaria). PloS one 3 (2008): e3222. 
15. Kitahara MV, Cairns SD, Stolarski J, et al. A comprehensive phylogenetic analysis of the Scleractinia (Cnidaria, Anthozoa) based on mitochondrial CO1 sequence data. PloS one 5 (2010): e11490.

16. Keshavmurthy S, Yang SY, Alamaru A, et al. DNA barcoding reveals the coral "laboratory-rat", Stylophora pistillata encompasses multiple identities. Scientific reports 3 (2013).

17. Veron JEN. New species described in Corals of the World, Australian Institute of Marine Science (2000).

18. Fukami H, Budd AF, Paulay G, et al. Conventional taxonomy obscures deep divergence between Pacific and Atlantic corals. Nature 427 (2004): 832-835.

19. Thompson JD, Higgins DG, Gibson TJ. CLUSTAL W: improving the sensitivity of progressive multiple sequence alignment through sequence weighting, position-specific gap penalties and weight matrix choice." Nucleic acids research 22 (1994): 4673-4680.

20. Tamura K, Stecher G, Peterson D, et al. MEGA6: Molecular Evolutionary Genetics Analysis version 6.0." Molecular biology and evolution 30 (2013): 2725-2729.

21. McMillan J, Mahony T, Veron JEN, et al. Nucleotide sequencing of highly repetitive DNA from seven species in the coral genusAcropora (Cnidaria: Scleractinia) implies a division contrary to morphological criteria. Marine Biology 110 (1991): 323-327.

22. Miller DJ, Harrison PL, Mahony TJ, et al. Nucleotide sequence of the histone gene cluster in the coral Acropora formosa (Cnidaria; Scleractinia): features of histone gene structure and organization are common to diploblastic and triploblastic metazoans. Journal of Molecular Evolution 37 (1993): 245-253.

23. Lopez JV, Knowlton N. Description of Montastrea coral sibling species with multiple genetic loci. Proceedings of the 8th International Coral Reef Symposium 2: 1613-1618.

24. Hunter CL, Morden CW, Smith CM. The utility of ITS sequences in assessing relationships among zooxanthellae and corals. Proceedings of the 8th International Coral Reef Symposium 2 (1998): 1599-1602.

25. Harrison PL, Wallace CC. Reproduction, dispersal and recruitment of scleractinian corals. In: Ecosystems of the World 25: Coral Reef (1990): 133-206.

26. Fadlallah YH. Sexual reproduction, development and larval biology in scleractinian corals. Coral reefs 2 (1983): 129-150.

27. Stolarski J, Kitahara MV, Miller DJ, et al. The ancient evolutionary origins of Scleractinia revealed by azooxanthellate corals." BMC evolutionary biology 11 (2011): 316.

28. Budd AF, Fukami H, Smith ND, et al. Taxonomic classification of the reef coral family Mussidae (Cnidaria: Anthozoa: Scleractinia). Zoological Journal of the Linnean Society 166 (2012): 465-529.

29. Dai Cf, Horng S. Scleractinia Fauna of Taiwan: Complex group (2009).

30. Kitano YF, Benzoni F, Arrigoni R, et al. A phylogeny of the family Poritidae (Cnidaria, Scleractinia) based on molecular and morphological analyses. PLoS One 9 (2014): e98406.

31. Robitzch V, et al. Absence of genetic differentiation in the coral Pocillopora verrucosa along environmental gradients of the Saudi Arabian Red Sea. Frontiers in Marine Science 2 (2015): 5. 
32. Benzoni F, Stefani F. Porites fontanesii, a new species of hard coral (Scleractinia, Poritidae) from the southern Red Sea, the Gulf of Tadjoura, and the Gulf of Aden, and its phylogenetic relationships within the genus. Zootaxa 3447 (2012): 56-68.

33. Mangubhai S, Souter P, Grahn M. Phenotypic variation in the coral Platygyra daedalea in Kenya: morphometry and genetics. Marine Ecology Progress Series 345 (2007): 105-115.

34. Miller K. Morphological variation in the coral genus Platygyra: environmental influences and taxonomic implications." Marine Ecology-Progress Series 110 (1994): 19-19.

Citation: Mohammed Ahmed Sadek, Mohammed Ismail Ahmed, Fedekar Fadel Madkour, Mahmoud Hasan Hanafy. Using DNA Barcoding Technique to Identify Some Scleractinian Coral Species along the Egyptian Coast of the Red Sea and Southern of Arabian Gulf. Journal of Environmental Science and Public Health 2 (2018): 52-63.

(C) 9 This article is an open access article distributed under the terms and conditions of the
Creative Commons Attribution (CC-BY) license 4.0 